\title{
10. Umwelt und Entwicklung
}

Christoph Stamm and Catherine Schümperli Younossian

\section{(2) OpenEdition \\ Journals}

Electronic version

URL: http://journals.openedition.org/sjep/224

DOI: $10.4000 /$ sjep.224

ISSN: 1663-9677

\section{Publisher}

Institut de hautes études internationales et du développement

\section{Printed version}

Date of publication: 1 avril 2004

Number of pages: $181-200$

ISSN: 1660-5926

\section{Electronic reference}

Christoph Stamm und Catherine Schümperli Younossian, «10. Umwelt und Entwicklung »,

Schweizerisches Jahrbuch für Entwicklungspolitik [Online], 23-1 | 2004, Online erschienen am: 10 August 2010, abgerufen am 08 September 2020. URL : http://journals.openedition.org/sjep/224 ; DOI :

https://doi.org/10.4000/sjep.224 


\section{Umwelt und Entwicklung*}

I M JAHR 2003 wurde der erste schweizerische Monitoringbericht über den Stand der nachhaltigen Entwicklung in der Schweiz veröffentlicht ${ }^{1}$. Aus dem Bericht wird ersichtlich, dass es der Schweiz in den letzten Jahren nicht gelungen ist, die Weichen in Richtung nachhaltige Entwicklung zu stellen. Vor allem die Umsetzung des Umweltaspektes der nachhaltigen Entwicklung kann zur Zeit weder in der Bevölkerung, noch in Bundesregierung oder -parlament auf breite Unterstützung zählen².

Der Schutz des Weltklimas stellt einen Bereich der nachhaltigen Entwicklung dar. Die internationale Gemeinschaft tut sich weiterhin schwer bei der Entwicklung und Umsetzung von allgemeingültigen Normen, welche die klimaerwärmenden Treibhausgasemissionen wirksam eindämmen. Daher ist das In-KraftTreten des Kyoto-Protokolls weiterhin gefährdet. Das Protokoll wurde im Juli 2003 von der Schweiz ratifiziert. Damit die eingegangenen Verpflichtungen zum Klimaschutz fristgerecht umgesetzt werden können, muss die Schweiz ihre Anstrengungen in den nächsten Jahren verstärken.

Das Jahr 2003 war von der Generalversammlung der UNO zum Internationalen Jahr des Wassers erklärt worden. Auf internationaler Ebene fand das 3. Weltwasserforum in Kyoto statt. In der Schweiz wurde die Bevölkerung mit zahlreichen Projekten für eine ganzheitliche Betrachtungsweise der Wasserthematik sensibilisiert.

Ein wichtiges Ereignis betreffend die Erhaltung der biologischen Vielfalt war das In-Kraft-Treten des Protokolls von Cartagena. Mit dem Protokoll über biologische Sicherheit wird erstmals der internationale Handel mit lebenden gentechnisch veränderten Organismen geregelt.

\subsection{Nachhaltige Entwicklung in der Schweiz}

Anlässlich der UNO-Konferenz über Entwicklung und Umwelt von 1992 in Rio de Janeiro und der UNO-Konferenz über Nachhaltige Entwicklung von 2002 in Johannesburg hat sich die Schweiz zusammen mit 178 weiteren Staaten verpflichtet, auf nationaler und internationaler Ebene eine Politik der nachhaltigen Entwicklung auszuarbeiten und umzusetzen. Seit 1999 gründet die Verpflichtung zur Nachhaltigkeit auf dem Artikel Nr. 73 der schweizerischen Bundesverfassung: „Bund und Kantone streben ein auf Dauer ausgewogenes Verhältnis zwischen der Natur und ihrer Erneuerungsfähigkeit einerseits und

* Teil 10.1, 10.2, 10.3 und 10.5 von Christoph Stamm, Politologe.

Teil 10.4 von Catherine Schümperli Younossian, Forschungsbeauftragte am iuéd.

1 Bundesamt für Statistik, ARE, BUWAL, Nachhaltige Entwicklung in der Schweiz - Indikatoren und Kommentare und MONET Schlussbericht - Methoden und Resultate, Neuenburg, November 2003.

2 Beispiele dafür sind die Ablehnung der Atomausstiegsintitiative, die Strassenbauprojekte des Parlaments sowie die überdurchschnittlichen Budgetkürzungen im Bundesamt für Umwelt Wald und Landschaft. 
ihrer Beanspruchung durch den Menschen anderseits an.“ Als Umsetzungshilfe dient die im Frühjahr 2002 verabschiedete „Strategie Nachhaltige Entwicklung“. Sie gibt die inhaltlichen und vorgehensbezogenen Rahmenbedingungen der bundesrätlichen Politik der nachhaltigen Entwicklung bis ins Jahr 2007 vor ${ }^{3}$. Neben konzeptionellen Leitlinien enthält die Strategie in zehn Handlungsfeldern insgesamt 22 wirkungsorientierte Massnahmen. Koordiniert wird die nationale Politik der Nachhaltigkeit vom Bundesamt für Raumentwicklung (ARE) und vom Interdepartementalen Ausschuss Rio (IDARio).

Im Rahmen des Entlastungsprogramms beschloss das Parlament im Herbst 2003, das Budget des Bundesamtes für Umwelt, Wald und Landschaft (BUWAL) bis 2006 um 104 Millionen Franken oder 15 Prozent zu kürzen ${ }^{4}$. Die Folgen sind laut BUWAL der Abbau von rund 20 Stellen sowie markante Einsparungen im Forstbereich, im Natur- und Landschaftsschutz sowie beim Vollzug der Umweltgesetzgebung. Die Kürzungen bedeuten eine Schwächung des Umweltaspektes der nachhaltigen Entwicklung in der Schweiz.

[Dahrbuch 2003, Nr. 1, in Kap. 10.1, „Neue Strategie 2002 für die nachhaltige Entwicklung“, S. 171-173.

\section{$\square$ Messung nachhaltiger Entwicklung}

Seit 2000 ist im Projekt MONET (Monitoring der Nachhaltigen Entwicklung) ein Indikatorensystem zur umfassenden Beobachtung der nachhaltigen Entwicklung der Schweiz entwickelt worden ${ }^{5}$. Mit der Veröffentlichung der Publikationen „Nachhaltige Entwicklung in der Schweiz - Indikatoren und Kommentare“ sowie „MONET Schlussbericht - Methoden und Resultate“ wurde das Projekt im November 2003 vorgestellt.

Mit Hilfe von 120 Indikatoren in 26 Teilbereichen werden Aussagen über die aktuelle Lage und Entwicklung der Schweiz hinsichtlich der sozialen, wirtschaftlichen und ökologischen Aspekte der nachhaltigen Entwicklung gemacht. Das Monitoringinstrument erlaubt eine regelmässige Berichterstattung über Stand und Verlauf der nachhaltigen Entwicklung in der Schweiz und erleichtert den internationalen Vergleich.

Da sich die drei verwendeten Zielbereiche ,gesellschaftliche Solidarität“, ,wirtschaftliche Leistungsfähigkeit“ und „ökologische Verantwortung" teilweise widersprechen, standen die Autoren bei der Erarbeitung des Berichts vor einer fast unlösbaren Aufgabe. Bei vielen Indikatoren ist nämlich unklar, ob eine Veränderung zu mehr oder zu weniger nachhaltiger Entwicklung führt. Daher fehlt bei manchen Indikatoren eine Evaluation, bei anderen ist diese fragwürdig. Die Schwierigkeit, eine Gesamtevaluation durchzuführen, mag mit ein Grund sein, weshalb die Autorinnen und Autoren auf eine Quantifizierung und auf einen Sammelindex der nachhaltigen Entwicklung verzichtet haben. Die Leserinnen und Leser sind damit aufgefordert, sich aus der Vielfalt und Widersprüchlichkeit der einzelnen Entwicklungen selbst ein Gesamtbild zu machen.

Bildet man sich ein Gesamturteil, so folgt der Schluss, dass sich die Schweiz nicht auf dem Pfad der nachhaltigen Entwicklung befindet und auf Kosten kom-

\footnotetext{
Bundesrat, Strategie Nachhaltige Entwicklung 2002, Bern, 27. März 2002.

Pressemitteilung BUWAL, Entlastungsprogramm: Folgen für das BUWAL, Bern, 2. Oktober 2003. MONET wird gemeinsam vom Bundesamt für Statistik (BFS), vom Bundesamt für Umwelt, Wald und Landschaft (BUWAL) sowie vom Bundesamt für Raumentwicklung (ARE) getragen.
} 
mender Generationen lebt. Die Autorinnen und Autoren diagnostizieren unter anderem einen Anstieg der Armut, einen exzessiven Verbrauch von Energie und natürlichen Ressourcen sowie eine Zunahme der radioaktiven Abfälle. Auch was die internationale Solidarität der Schweiz angeht, ist die Bilanz laut Indikatorenmodell tendenziell negativ: Die Schweiz gefährdet mit ihrem $\mathrm{CO}_{2}$-Ausstoss das Weltklima; die öffentlichen Gelder für Entwicklungszusammenarbeit stagnieren; die Beiträge der Entwicklungszusammenarbeit für die ärmsten Länder haben im letzten Jahrzehnt abgenommen und nur eine Minderheit der Schweizer Bevölkerung spricht sich für eine grössere Solidarität mit der Bevölkerung der Entwicklungsländer aus.

\section{$\square$ Nachhaltigkeitsziele der Umweltorganisationen}

Die schweizerischen Umweltorganisationen wollen den Umsetzungsprozess der Nachhaltigkeitsstrategie des Bundesrates aktiv mitgestalten und den Stellenwert des Umweltaspekts der nachhaltigen Entwicklung erhöhen. Dazu legten sie im November 2003 ein Dokument vor, das die Ziele definiert, welche die Schweiz bis zum Jahr 2012, das heisst 20 Jahre nach dem Erdgipfel von Rio, erreichen sollte $^{6}$. Ein Kapitel ist dabei der Umwelt-Aussenpolitik gewidmet. Darin befinden die Umweltorganisationen, dass die Industrieländer durch ihre Produktionsweise und ihr Konsumverhalten die Hauptverursacher der globalen Umweltzerstörung sind, dass aber die Auswirkungen dieses Verhaltens vor allem die Entwicklungsländer tragen müssen. „Dies bedeutet, dass die Industrieländer einerseits ihr Konsumverhalten ändern müssen, um weniger Ressourcen zu beanspruchen. Andererseits müssen die Entwicklungsländer bei ihrer Entwicklung durch Technologietransfer und finanzielle Mittel massiv unterstützt werden." 7 Konkret fordern die Umweltorganisationen, dass die Gelder für Entwicklungszusammenarbeit erhöht und für den Erhalt und die nachhaltige Nutzung natürlicher Ressourcen eingesetzt werden. Umweltabkommen und das Recht auf Nahrung sollen Vorrang vor den WTO-Regeln erhalten; IWF und Weltbank sollen bei ihrer Arbeit Nachhaltigkeitskriterien gerecht werden. Ausserdem fordern die Umwelt-NRO, dass Unternehmen mit Sitz in der Schweiz auch an Produktionsstandorten im Ausland die in der Schweiz geltenden Umweltstandards einhalten.

\section{QUELLEN}

Bundesrat, Strategie Nachhaltige Entwicklung 2002, Bern, 27. März 2002.

BUWAL, Umwelt Schweiz, Band 1 und 2, Bern, 2002.

Bundesamt für Statistik, ARE, BUWAL, Nachhaltige Entwicklung in der Schweiz - Indikatoren und Kommentare, Neuenburg, November 2003.

Bundesamt für Statistik, ARE, BUWAL, MONET Schlussbericht - Methoden und Resultate, Neuenburg, November 2003.

Equiterre, Greenpeace Schweiz, Pro Natura, VCS, WWF Schweiz, „Umweltziele 2012 - Die Schweiz ökologisch erneuern“, Bern, November 2003.

\section{INTERNET-ADRESSEN}

Bundesamt für Raumentwicklung (ARE) : <www.are.ch>.

Bundesamt für Umwelt, Wald und Landschaft (BUWAL) : <www.buwal.ch $>$.

6 Equiterre, Greanpeace Schweiz, Pro Natura, VCS, WWF Schweiz, Umweltziele 2012 - Die Schweiz ökologisch erneuern, Bern, November 2003.

7 ibd., Seite 18. 
Im Jahr 2002 hatte der Weltgipfel zur Nachhaltigen Entwicklung in Johannesburg stattgefunden. An der Konferenz wurden keine signifikanten Fortschritte erzielt ${ }^{8}$. Der verabschiedete Umsetzungsplan wiederholt grossenteils die anlässlich früherer Konferenzen geäusserten Absichten. Auch wurden keine neuen Mittel zur Verwirklichung der Entwicklungsziele gesprochen. Im Jahr 2003 ging es nun um die weitere Umsetzung der am Gipfel bestätigten Ziele.

Jahrbuch 2003, Nr. 1, Kap. 10.2., ,Weltgipfel für Nachhaltige Entwicklung“.

\section{$\square$ UNO-Kommission für nachhaltige Entwicklung}

Vom 28. April bis 9. Mai 2003 trat die UNO-Kommission für nachhaltige Entwicklung (CSD) zu ihrem ersten Jahrestreffen in New York nach dem Nachhaltigkeitsgipfel in Johannesburg zusammen ${ }^{9}$. Auf dem Programm standen die Reform der Arbeitsweise und die Festlegung der Schwerpunktthemen für die nächsten Jahre.

Das 1992 anlässlich des Erdgipfels von Rio gegründete UNO-Gremium begleitet und überwacht die Umsetzung der Verpflichtungen der internationalen Gemeinschaft im Bereich der nachhaltigen Entwicklung.

Die Kommission entschied, künftig Umsetzungszyklen auf einer Zweijahresbasis durchzuführen. Das erste Jahr dient der Überprüfung der Umsetzung der nachhaltigen Entwicklung und dem Austausch konkreter Erfahrungen im Umsetzungsprozess; im zweiten Jahr sollen politische Massnahmen und Empfehlungen ausgehandelt und beschlossen werden.

Im zukünftigen Arbeitsprogramm werden die so genannten „WEHAB“-Themen $^{10}$ - Wasser, Energie, Gesundheit, Landwirtschaft und biologische Vielfalt sowie diejenigen Bereiche im Vordergrund stehen, für die es noch keine zufriedenstellende internationale Regulierung oder zuständige Institution gibt. In der ersten Zweijahresperiode 2004-2005 bilden die Themen Wasser, sanitäre Anlagen und menschliche Siedlungen den Schwerpunkt.

Die Kommission beschloss ausserdem, Erfahrungen, Kenntnisse und Ressourcen von regionaler Ebene, internationalen Institutionen, Nichtregierungsorganisationen und der Privatwirtschaft besser zu nutzen und Organisationen aktiver in die Kommissionsarbeit einzubeziehen.

Die Schweizer Delegation zeigte sich über das elfte Jahrestreffen zufrieden, war sie doch eine der Initianten der beschlossenen Kommissionsreform ${ }^{11}$.

8 Einziges neues wichtiges Zeitziel ist die Halbierung der Anzahl Menschen ohne Zugang zu Abwasserentsorgung bis zum Jahr 1015.

9 Commission on Sustainable Development, 11th Session : <www.un.org/esa/sustdev/csd/csd11/CSD11. htm>.

10 Um einer Überfrachtung des Nachhaltigkeitsgipfel von Johannesburg zu entgehen, stellten die Regierungen auf Vorschlag von UNO-Generalsekretär Kofi Annan fünf Bereiche in den Mittelpunkt der Verhandlungen. WEHAB steht damit für „Water, Energy, Health, Agriculture, Biodiversity“.

11 Le Temps, „La Suisse remporte une victoire diplomatique à l'ONU“, 12. Mai 2003, und Pressemitteilung BUWAL, 25. April 2003. 


\section{$\square$ Förderung nachhaltiger Produktions- und Konsumweisen}

In dem am Nachhaltigkeitsgipfel in Johannesburg verabschiedeten Umsetzungsplan wird deutlich auf die Problematik der nicht nachhaltigen Produktions- und Konsumweisen hingewiesen: „Um eine nachhaltige Entwicklung zu gewährleisten, ist es unumgänglich, dass die Gesellschaften ihre Art und Weise, wie sie produzieren und konsumieren, radikal modifizieren."12

Schätzungen zufolge liegt die aktuelle weltweite Produktions- und Konsumhöhe bereits 25 Prozent und diejenige der Länder mit hohen Einkommen 160 Prozent über der ökologischen Tragfähigkeit der Erde.

Um einen Beitrag zur internationalen Förderung nachhaltiger Produktions- und Konsumweisen zu leisten, trafen sich Experten aus sechzig Ländern vom 16. bis 19. Juni zu einer Konferenz in Marrakesch ${ }^{13}$. An dem von der UNO-Hauptabteilung für wirtschaftliche und soziale Angelegenheiten (UN/DESA) und dem Umweltprogramm der Vereinten Nationen (UNEP) organisierten Treffen wurde ein Zehnjahresplan ausgearbeitet. Dieser ist dazu bestimmt, die Produktionsund Konsumweisen $\mathrm{zu}$ überdenken und die internationalen Verpflichtungen betreffend die Einführung nachhaltiger Lebensweisen zu konkretisieren. Die im neuen „Marrakesch-Prozess“ angestrebten Strategien sollen helfen, ein internationales Regelwerk aufzubauen, wirtschaftliche Anreize zu schaffen sowie Partnerschaften zwischen Regierungsstellen, internationalen Organisationen, Privatwirtschaft und Nichtregierungsorganisationen zu etablieren.

\section{QUELLE}

Martens Jens, Sterk Wolfgang, Multilateralismus zwischen Blockadepolitik und Partnerschaftsrhetorik Eine Bilanz des Gipfels von Johannesburg, weed Arbeitspapier, Bonn : September, 2002.

\section{INTERNET-ADRESSE}

UN Departement of Economic and Social Affairs, Division for Sustainable Development: <www.un.org/ esa/sustdev>.

\subsection{Umsetzung der Rahmenkonvention über Klimaänderungen}

Die 1994 in Kraft getretene Klimakonvention hat zum Ziel, die Treibhausgasemissionen in der Atmosphäre auf einem Niveau zu stabilisieren, das eine Veränderung des Klimasystems verhindert.

Das menschenverursachte Kohlendioxid ist mengenmässig das wichtigste Treibhausgas und damit hauptverantwortlich für globale Klimaerwärmung. Daher müsste für einen wirksamen Klimaschutz in erster Linie der Verbrauch der Kohlendioxid produzierenden fossilen Energieträger reduziert werden.

Laut Schätzungen haben jedoch im Jahr 2002 die weltweiten energiebedingten Kohlendioxidemissionen gegenüber dem Vorjahr um 3 Prozent zugenommen ${ }^{14}$. Die Emissionen der Industrieländer blieben konstant, während die Entwick-

12 Plan of Implementation of the World Summit on Sustainable Development, Johannesburg, 2002.

13 UN Department of Economic and Social Affairs, Division for Sustainable Development, Media Service : <www.un.org/esa/sustdev/media/media.htm>.

14 Seit 1990 beträgt die kumulierte Zunahme 13 Prozent. Germanwatch, Ludwig-Bölkow-Systemtechnik $\mathrm{GmbH}$, Analysis of BP Statistical Review of World Energy with respect to $\mathrm{CO}_{2}$ emissions, Bonn, Juli 2003. 
lungsländer eine Zunahme um 7 Prozent verzeichneten. Die Stabilisierung der Emissionen in den reichen Staaten ist mehrheitlich auf geringes Wirtschaftswachstum und Deindustrialisierung und weniger auf eine effiziente Klimapolitik zurïckzuführen. Unter den westlichen Industriestaaten befinden sich lediglich Deutschland, Grossbritannien und Schweden auf „Klimaschutzkurs“. Diese Länder haben ihre energiebedingten Kohlendioxidemissionen in den letzten zwölf Jahren um 7 bis 13 Prozent reduziert.

In Chicago hat im Dezember 2003 eine Umweltbörse ihren Handel aufgenommen $^{15}$. Gehandelt werden am Chicago Climate Exchange verbriefte Emissionsrechte, so genannte $\mathrm{CO}_{2}$-Zertifikate. Die 34 Teilnehmer haben sich freiwillig Reduktionsziele für den Ausstoss von $\mathrm{CO}_{2}$ auferlegt. Wer sauberer produziert, kann Zertifikate abgeben. Wer es nicht schafft, die gesteckten Ziele zu erreichen, muss Zertifikate dazukaufen. Mit der weltweit ersten Börse für Emissionshandel sollen wertvolle Erfahrungen zur Verwendung marktwirtschaftlicher Instrumente für die Lösung von Umweltproblemen gesammelt werden. Auch in der EU laufen die Vorbereitungen für ein Handelssystem für Treibhausgasemissionen ${ }^{16}$. Aufgrund des Kyoto-Protokolls werden die europäischen Unternehmen zur Teilnahme am Handel verpflichtet werden.

\section{$\square$ Ratifizierung des Kyoto-Protokolls}

Das 1997 im Rahmen der Klimakonvention verabschiedete Protokoll von Kyoto verpflichtet die traditionellen Industriestaaten ${ }^{17}$, ihre Treibhausgasemissionen bis zum Zeitraum 2008-2012 gegenüber 1990 um mindestens 5,2 Prozent zu reduzieren ${ }^{18}$.

Von diesen im Annex I aufgeführten Staaten hatten Ende 2003 nur die USA, Russland, Australien, Monaco und Liechtenstein das Klimaschutzprotokoll von Kyoto nicht ratifiziert. Ohne die Ratifizierung der USA oder Russlands kann das Protokoll jedoch nicht in Kraft treten. Nachdem im Jahr 2002 viele Beobachter davon ausgegangen waren, dass Russland das Protokoll in absehbarer Zeit ratifizieren würde, näherte sich das Land im Jahr 2003 der Position der USA und Australiens an und beurteilte das Protokoll als zu kostspielig und ineffizient. Die USA sind der Ansicht, dass das Protokoll ihrer wirtschaftlichen Entwicklung im Wege steht und dass sie bei einer Ratifizierung gegenüber den Schwellenländern im Nachteil wären ${ }^{19}$. Länder wie China, Indien, Südkorea, Mexiko und Brasilien, deren $\mathrm{CO}_{2}$-Ausstoss sich in den letzten zwölf Jahren teilweise massiv erhöht hat und die zu bedeutenden $\mathrm{CO}_{2}$-Produzenten geworden sind, werden durch das Kyoto-Protokoll nicht zu Emissionsreduktionen verpflichtet. Die USA fordern deshalb neue Klimaverhandlungen, bei denen auch die Schwellenländer einen Beitrag zum Klimaschutz leisten müssen.

$15 \quad$ NZZ am Sonntag, 30. November 2003.

16 Emissions Trading: <http://europa.eu.int/comm/environment/climat/emission.htm>.

17 Es handelt sich hauptsächlich um die OECD-Staaten (ohne Mexiko, Korea und die Türkei) sowie um die Staaten des ehemaligen Ostblocks (ohne Weissrussland, Kasachstan und Usbekistan).

$18 \mathrm{Zu}$ den Treibhausgasen gehören die Emissionen aus fossilen Energieträgern und aus der Entwaldung sowie Prozess-Emissionen $\left(\mathrm{CO}_{2}\right)$, Methan (CH4), Lachgas (N2O), teilhalogenierte Fluor-Kohlenwasserstoffe (HFC), perfluorierte Kohlenwasserstoffe (PFC), Schwefelhexafluorid (SF6).

19 George Bush „President Bush Discusses Global Climate Change“, Juni 2001 : <www.whitehouse. gov/news/releases/2001/06/20010611-2.html>. 
Die Frage ist nun, ob diejenigen Länder, die das Kyoto-Protokoll ratifiziert haben, mit dessen Umsetzung weiterfahren, als sei es in Kraft, oder ob sie, von den Freeridern geschwächt, mit ihren Anstrengungen zum Klimaschutz nachlassen.

\section{$\square$ Neunte Vertragsparteienkonferenz in Mailand}

Vom 1. bis 12. Dezember 2003 trafen sich die 188 Vertragsparteien der Klimakonvention sowie über 300 internationale Organisationen und Nichtregierungsorganisationen zur neunten Klimakonferenz (COP-9) in Mailand ${ }^{20}$. Angesichts neuer Klimadaten der Weltorganisation für Meteorologie (WMO) sowie der Hitzewellen, Dürren und verheerenden Überschwemmungen des Jahres 2003 waren sich die Delegierten weitgehend einig, dass der Klimawandel eine der wichtigsten globalen Herausforderungen darstellt. Keine Einigung bestand aber weiterhin über den zu gehenden Weg, um dem Klimawandel entgegenzutreten. Die Befürworter des Kyoto-Protokolls wollen den Ausstoss der Treibhausgase verringern; die Gegner setzen auf die „Entsorgung“ oder Speicherung von $\mathrm{CO}_{2}$ unter dem Boden, und die am meisten betroffenen Länder fordern Gelder für die Anpassung an den Klimawandel.

Der grösste Fortschritt wurde bei der Einigung zur Frage der Kohlenstoffsenken $^{21}$ im Forstsektor des Clean Development Mechanism (CDM) erzielt ${ }^{22}$. Die Delegierten legten die Bedingungen fest, unter denen die Industrieländer Aufforstungsprojekte in Entwicklungsländern finanzieren können, um ihre Treibhausgasemissionen zu kompensieren. Die Schweiz setzte sich dafür ein, dass die Senken im Forstsektor gewissen Kriterien bezüglich Umwelt, Biodiversität und der Rechte der lokalen Bevölkerung genügen. Monokulturplantagen sind jedoch zugelassen. Mit der Einigung in der Frage der Senken ist der CDM operationalisiert ${ }^{23}$. Dank der Bereinigung weiterer Detailfragen steht der Umsetzung des Kyoto-Protokolls rein organisatorisch nichts mehr im Wege.

An der Konferenz ging es ferner um die Ausgestaltung des Fonds für die am wenigsten entwickelten Länder und des Special Climate Change Fund. Die EU, Kanada, Neuseeland, Island, Norwegen und die Schweiz erneuerten ihr Versprechen, ab 2005 jährlich 410 Millionen US-Dollar für die Fonds und die Entwicklungsländer bereitzustellen ${ }^{24}$.

Mit der Unterstützung der Schweiz wurde entschieden, dass die Umsetzung der Klimakonvention nach 2012 in Angriff genommen wird. Damit werden Arbeiten hinsichtlich Massnahmen aufgenommen, welche über das Kyoto-Protokoll hinausgehen.

20 9. Vertragsparteienkonferenz: <http://unfccc.int/cop9>; Pressemitteilung BUWAL, 11. Dezember 2003; Neue Zürcher Zeitung, 11./12. Dezember 2003; International Institute for Sustainable Development, Earth Negotiations Bulletin, Summary of the Ninth Conference of the Parties to the UN Framework Convention on Climate Change, 15. Dezember 2003

21 Als Senken bezeichnet man Flächen, zum Beispiel Wälder, die der Atmosphäre Treibhausgase entziehen.

22 Der Clean Development Mechanism (Mechanismus für umweltgerechte Entwicklung) gehört zusammen mit dem internationalen Emissionshandel und dem Joint Implementation Mechanism (Mechanismus für gemeinsame Umsetzung) zu den drei flexiblen Mechanismen, deren sich die Industrieländer zur Einhaltung der Verpflichtungen des Kyoto-Protokolls bedienen können.

23 Es fehlen noch die Modalitäten für Prozeduren bei Projekten mit kleinen Kohlenstoffsenken.

24 Pressemitteilung UNFCCC, Milan Conference concludes as ministers call for urgent and coordinated action to climate change, Mailand, 12. Dezember 2003. 


\section{Der Clean Development Mechanism als Instrument der internationalen Entwicklungszusammenarbeit}

1a) Industrieländer - der Staat oder Privatunternehmen - investieren in emissionsreduzierende Projekte in Entwicklungsländern.

1b) Entwicklungsländer - der Staat oder Privatunternehmen - führen selbst Projekte durch, welche zur Reduktion der Treibhausgasemissionen beitragen.

2) Dadurch wird im Entwicklungsland ein bestimmter Sektor modernisiert und es entsteht ein positiver Beitrag zum globalen Klimaschutz.

3a) Das investierende Industrieland kann sich die durch seine Investitionen im Entwicklungsland erzielten Emissionsreduktionen auf seine Verpflichtungen anrechnen.

3b) Das Entwicklungsland kann die erzielten Emissionsgutschriften an Industrieländer verkaufen.

Quelle: Deutsche Gesellschaft für Technische Zusammenarbeit, Clean Development Mechanism, <www.gtz.de/climate>.

Anlässlich der Klimakonferenz wurde in Genf eine Investitionsplattform für Klimaschutzprojekte (Climate Investment Partnership, CIP) lanciert. Die von der Schweiz mit 300'000 Franken unterstützte Plattform bringt Investoren und Entwickler von Klimaschutzprojekten zusammen und soll die Nutzung der flexiblen Mechanismen vereinfachen ${ }^{25}$.

\section{$\square$ Klimapolitik der Schweiz}

Verpflichtung zum Klimaschutz und Entwicklung der Treibhausgasemissionen

Für die schweizerische Klimapolitik sind das nationale $\mathrm{CO}_{2}$-Gesetz und das Kyoto-Protokoll von zentraler Bedeutung. Als eines der letzten Industrieländer hinterlegte die Schweiz am 9. Juli 2003 bei den Vereinten Nationen in New York die Ratifikationsurkunde zum Kyoto-Protokoll und trat dem im Rahmen der Klimakonvention geschaffenen Vertragswerk bei. Die Schweiz verpflichtet sich damit, ihren Ausstoss von Treibhausgasen bis zum Zeitraum 2008-2012 gegenüber dem Stand von 1990 um 8 Prozent zu senken.

Das nationale $\mathrm{CO}_{2}$-Gesetz verlangt bis 2010 eine Senkung der $\mathrm{CO}_{2}$-Emissisonen um 10 Prozent unter das Niveau von 1990. Das Gesetz beschränkt sich auf die Kohlendioxidemissionen, welche auf die energetische Nutzung fossiler Brenn- und Treibstoffe zurückzuführen sind. Diese stellen drei Viertel der im Kyoto-Protokoll definierten Treibhausgase dar, was den hohen Stellenwert des $\mathrm{CO}_{2}$-Gesetzes für die Einhaltung des Kyoto-Protokolls unterstreicht.

Der Ausstoss der im Kyoto-Protokoll aufgeführten Treibhausgase blieb in der Schweiz zwischen 1990 und 2001 mit einer Zunahme von 0,7 Prozent praktisch stabil und belief sich im Jahr 2002 auf 53 Millionen Tonnen $\mathrm{CO}_{2}$ - ̈̈quivalente ${ }^{26}$.

Die $\mathrm{CO}_{2}$-Emissionen gemäss $\mathrm{CO}_{2}$-Gesetz nahmen 2002 gegenüber 2001 um 1,4 Prozent $a b^{27}$. Die Ursachen der Abnahme waren höhere Wintertemperaturen, eine rückläufige Industrieproduktion und die Wirkung des Programms EnergieSchweiz.

25 Pressemitteilung seco, Klimaschutzprojekte im Rahmen des Kyoto-Protokolls, Bern, 3. Dezember 2003. Siehe auch Climate Investment Partnership: <www.climateinvestores.com>.

26 BUWAL, Treibhausgasinventar der Schweiz, Stand Mai 2003, Bern, 2003.

27 BUWAL, Emissionen nach $\mathrm{CO}_{2}$-Gesetz und Kyoto-Protokoll, Bern, August 2003. 


\section{SwissFlex und Register für Emissionsgutschriften und Transaktionen}

Für die Umsetzung und Betreuung der flexiblen Mechanismen des Kyoto-Protokolls wurde ein nationales Sekretariat namens SwissFlex gegründet. Es ist im BUWAL in der Sektion Ökonomie und Klima angesiedelt. Die Aktivitäten rund um die Umsetzung der flexiblen Mechanismen sowie die Prüfung und Genehmigung von Projektanträgen werden innerhalb der interdepartementalen Arbeitsgruppe IDA-SwissFlex koordiniert ${ }^{28}$.

Das Kyoto-Protokoll verpflichtet die Industriestaaten, ein nationales Register zur Erfassung aller Emissionsgutschriften und Transaktionen zu führen. Das Register ist eine Voraussetzung für die Teilnahme an den drei flexiblen Mechanismen. Das sich im Aufbau befindende schweizerische Register soll bis Ende 2005 betriebsbereit $\operatorname{sein}^{29}$.

\section{Wirkung des Energiesparprogramms}

Das Programm EnergieSchweiz konnte mit freiwilligen Fördermassnahmen seine Wirkung auf den Energieverbrauch und die $\mathrm{CO}_{2}$-Emissionen im Jahr 2002 gegenüber dem Vorjahr um 30 Prozent steigern. Die eingesetzten Mittel von Bund und Kantonen in Höhe von rund 111 Millionen Franken lösten Investitionen von rund 900 Millionen Franken aus. Die Einsparungen durch die freiwilligen und gesetzlichen Massnahmen werden auf 0,8 Prozent des gesamten Energieverbrauchs geschätzt. Ohne das Programm EnergieSchweiz und das Vorgängerprogramm Energie2000 lägen die Kohlendioxidemissionen 6 bis 9 Prozent über dem Niveau von 2003.

Im Rahmen des Entlastungsprogramms für das Bundesbudget schlug der Bundesrat dem Parlament eine Reduktion des Budgets von EnergieSchweiz von bisher 55 Millionen Franken auf 20 Millionen ab 2006 vor. Im Dezember 2003 folgte das Parlament nur teilweise den Wünschen des Bundesrates und kürzte das Energiesparprogramm um 10 Millionen auf 45 Millionen Franken. Die 10 Millionen Franken sollen hauptsächlich bei der Förderung energieeffizienter und innovativer Technologien eingespart werden ${ }^{30}$.

\section{Künftige Umsetzung der Verpflichtungen}

Es wird immer deutlicher, dass die freiwilligen Anstrengungen zur $\mathrm{CO}_{2}$-Reduktion nicht ausreichen, um den Verpflichtungen innerhalb der gesetzlichen Frist nachzukommen. Gelingt es nicht, mit freiwilligen Massnahmen das Ziel zu erreichen, kann der Bundesrat gemäss $\mathrm{CO}_{2}$-Gesetz ab 2004 eine steuerneutrale $\mathrm{CO}_{2}$-Abgabe von bis zu 50 Rappen pro Liter Treibstoff einführen. Die Einführung einer solchen Lenkungsabgabe wird von den Umweltverbänden für Mitte 2004 gefordert $^{31}$.

Der Bundesrat verzichtet auf eine rasche Einführung der $\mathrm{CO}_{2}$-Abgabe und möchte den freiwilligen Massnahmen trotz Budgetkürzungen mehr Zeit einräumen. Daher

28 Beteiligt an IDA-SwissFlex sind das BUWAL, das Bundesamt für Energie, das Staatssekretariat für Wirtschaft und die DEZA.

29 Ernst Basler+Partner, BUWAL, Nationales Register für Emissionsgutschriften und Transaktionen, Zollikon, 13. Mai 2003.

30 EnergieSchweiz, Entlastungsprogramm 2003, Bern, 3. Dezember 2003; siehe auch Rechsteiner Rudolf, ,7.9. EnergieSchweiz: Eine Trockenübung ?“, in Rechsteiner Rudolf, Grün gewinnt - Die letzte Ölkrise und danach, orell füssli Verlag AG, Zürich, 2003.

31 WWF-Schweiz, WWF und Wirtschaft machen Druck - Klimapetition, Bern, Oktober 2003. 
prüft er den Vorschlag eines Klimarappens der Erdöl-Vereinigung ${ }^{32}$. Mit einer freiwilligen Abgabe von einem Rappen pro Liter Benzin möchte die Erdöllobby eine Lenkungsabgabe verhindern. Der jährliche Ertrag des Benzinrappens von 60 Millionen Franken soll für $\mathrm{CO}_{2}$-reduzierende Massnahmen im In- und Ausland verwendet werden. 80 Prozent der $\mathrm{CO}_{2}$-Reduktionen würden durch den Kauf von Kyoto-Zertifikaten im Ausland realisiert. Dies würde aber der Botschaft des Bundesrates zur Ratifizierung des Kyoto-Protokolls widersprechen. Laut dieser sollen die zu erzielenden Emissionsreduktionen hauptsächlich in der Schweiz realisiert werden ${ }^{33}$.

\section{QUELLEN}

BUWAL, Umwelt Schweiz, Band 1 und 2, Bern, 2002.

BUWAL, Magazin Umwelt 2/2002, ,Klimawandel“, Bern, 2003.

International Institute for Sustainable Development, Earth Negotiations Bulletin, Summary of the Ninth Conference of the Parties to the UN Framework Convention on Climate Change, 15. Dezember 2003.

Rechsteiner Rudolf, Grün gewinnt - Die letzte Ölkrise und danach, orell füssli Verlag AG, Zürich, 2003.

\section{INTERNET-ADRESSEN}

Bundesamt für Umwelt, Wald und Landschaft (BUWAL) : <www.buwal.ch $>$.

Climate Action Network (CAN) : <www.climatenetwork.org $>$.

Forum Umwelt und Entwicklung : <www.forumue.de>

Germanwatch : <germanwatch.org $>$.

Intergovernmental Panel on Climate Change (IPCC) : $<$ www.ipcc.ch $>$.

International Institute for Sustainable Development (IISD) : <www.iisd.org $>$.

Ninth Session of the Conference of the Parties: <www.unfccc.int/cop9>.

United Nations Framework Convention on Climate Change (UNFCCC) : <www.unfccc.int $>$.

\subsection{Internationales Jahr des Süsswassers 2003}

2003 wurde von den Vereinten Nationen zum Internationalen Jahr des Süsswassers erklärt. Zahlreiche internationale und nationale Ereignisse prägten dieses Jahr. Zu erwähnen ist die Veröffentlichung eines Syntheseberichts über die Situation des Süsswassers in der Welt sowie die Organisation des 3. Weltwasserforums in Kyoto (Japan) im März 2003. Auch in der Schweiz wurden anlässlich des Internationalen Süsswasserjahres zahlreiche Projekte lanciert.

Die Wasserkrise auf unserem Planeten nimmt alarmierende Ausmasse an: Jeden Tag sterben 25'000 Kinder an durch verunreinigtes Trinkwasser verursachten Krankheiten. In der Landwirtschaft herrscht akuter Wassermangel. Auch zahlreiche Städte sind von Besorgnis erregender Versorgungsnot geplagt - ganz zu schweigen davon, dass Wasser in vielen Regionen der Welt zur Quelle von Spannungen wird ${ }^{34}$.

Mehrere internationale Konferenzen ${ }^{35}$ und auch das internationale Jahrzehnt für

32 Ein Expertenbericht für das BUWAL kam zum Schluss, dass sowohl ein Klimarappen als auch eine Kombination Klimarappen/ $\mathrm{CO}_{2}$-Abgabe das $\mathrm{CO}_{2}$-Reduktionsziel erreichen können. Medienmitteilung des BUWAL, 19. Juni 2003.

33 Bundesrat, Botschaft über das Protokoll von Kyoto zum Rahmenübereinkommen der VN über Klimaänderungen, Bern, August 2002 (BB1 2002 6385).

34 Weltwasserrat, Publication du rapport „Les actions pour l'eau dans le monde“, Pressemitteilung, 10. März 2003. 
Trinkwasser und Sanierung (1981-1991), eine wichtige Initiative der Vereinten Nationen, haben dafür gesorgt, dass das Thema Wasser in den Mittelpunkt des internationalen Interesses gerückt ist. Diese Initiativen gelten als Meilensteine in der Entwicklung einer neuen Art der Wasserbewirtschaftung, die neben den ökonomischen Zielsetzungen auch soziale und ökologische Anliegen berücksichtigt. Im Jahr 1996 wurde in Marseille der Weltwasserrat geschaffen, um die institutionelle Vernetzung der zahlreichen Stellen und Organisationen, die sich mit der Wasserbewirtschaftung befassen, sowie das Verständnis für die gemeinsamen Probleme und Anliegen zu fördern. Der Weltwasserrat soll insbesondere alle drei Jahre internationale Konferenzen zum Thema Wasser ausrichten. Das erste Weltwasserforum fand 1997 in Marrakesch, das zweite im Jahr 2000 in Den Haag und das dritte 2003 in Kyoto statt ${ }^{36}$.

Im Dezember 2000 beschloss die Generalversammlung der Vereinten Nationen, das Jahr 2003 zum Internationalen Jahr des Süsswassers zu erklären. Alle Länder und Städte wurden aufgefordert, Veranstaltungen zum Thema Wasser zu organisieren. Die von der Regierung Tadschikistans eingereichte Entschliessung wurde am 20. Dezember 2000 mit der Unterstützung von 148 Ländern verabschiedet. Darin werden die Regierungen, die Vereinten Nationen und alle übrigen Akteure aufgefordert, das Internationale Jahr des Süsswassers zum Anlass $\mathrm{zu}$ nehmen, um das Bewusstsein für die Bedeutung der nachhaltigen Nutzung, Bewirtschaftung und des Schutzes des Süsswassers zu stärken. Ausserdem werden die Regierungen, die nationalen und internationalen Organisationen, die Nichtregierungsorganisationen und der Privatsektor aufgefordert, freiwillige Beiträge zu leisten und die Umsetzung der Wasser-Agenda mit voranzutreiben.

Mit dem Internationalen Jahr des Süsswassers soll unter anderem ein in der Millenniums-Erklärung verankertes Ziel in Bezug auf das Wasser erreicht werden: den Anteil der Menschen, die ohne Wasserversorgung sind, bzw. sich diese nicht leisten können, bis 2015 halbieren und die nicht nachhaltige Nutzung von Wasserressourcen beenden. Das Ziel wurde anlässlich des Weltgipfels über die nachhaltige Entwicklung von Johannesburg im Jahr 2002 bekräftigt und um die Verpflichtung ergänzt, den Anteil der Menschen, die keinen Zugang zu einem Basisversorgungsnetz haben, bis 2015 um die Hälfte zu reduzieren. Dabei wurde die Schlüsselrolle des Wassers in den Bereichen Landwirtschaft, Energie, Gesundheit, Biodiversität und Ökosysteme sowie für die Armutsbekämpfung anerkannt.

Das Internationale Jahr des Süsswassers bietet die Chance, die Umsetzung der Grundsätze für eine integrierte Bewirtschaftung der Wasserressourcen zu beschleunigen, die bestehenden Tätigkeiten zu fördern und auf internationaler, regionaler und nationaler Ebene neue Initiativen bezüglich der Wasserressourcen zu ergreifen.

35 Wasserkonferenz der Vereinten Nationen, Mar del Plata (1977); Konferenz von Dublin über Wasser und Umwelt (1992); Erstes Weltwasserforum, Marrakesch (1997); Zweites Weltwasserforum, Den Haag (2000).

36 Siehe unten, 3. Weltwasserforum. 


\section{Weltwasserforum, März 2003}

Das 3. Weltwasserforum tagte vom 16. bis zum 23. März 2003 in Kyoto, Osaka und Shiga (Japan). Die UNO-Generalversammlung hatte den Weltwasserrat und die japanische Regierung mit der Organisation des internationalen Ereignisses beauftragt. An die 24'000 Personen aus 182 Ländern nahmen an der Konferenz teil - über dreimal mehr Teilnehmer, als ursprünglich geplant. Die Delegierten beteiligten sich an rund 350 verschiedenen Sitzungen zu 38 Themen im Zusammenhang mit dem Wasser. Gegenstand der Gespräche waren insbesondere die Befriedigung der steigenden Nachfrage nach Trinkwasser, die notwendige Verbesserung der Gesundheits- und Hygienebedingungen in der Nahrungsmittelproduktion sowie die Deckung der Bedürfnisse im Verkehrs-, Energieund Umweltschutzbereich. Dazu müssen die Länder eine effizientere Gouvernanz entwickeln, Kapazitäten ausbauen und über eine angemessene Finanzierung verfügen. Die Teilnehmer gingen am 3. Weltwasserforum über hundert neue Verpflichtungen für das Wasser ein ${ }^{37}$.

\section{Ministererklärung}

Nach den Diskussionen des 3. Weltwasserforums wurde eine Ministererklärung angenommen, die im Wesentlichen folgende Elemente umfasst:

- Wasser ist für die Entwicklung und für die Beseitigung von Armut und Hunger unverzichtbar; dieser Frage muss unbedingt Priorität eingeräumt werden.

$\checkmark$ Die benötigten finanziellen Mittel sollen namentlich durch die Anwendung des „Verursacherprinzips“ beschafft werden.

口 Private Investitionen sind erforderlich, dürfen jedoch die öffentliche Kontrolle über die Wasservorkommen nicht beeinträchtigen.

- Die öffentliche Entwicklungshilfe im Bereich Wasser soll bis zum Jahr 2015 verdoppelt werden.

๑ Die Anrainerstaaten von Wasserläufen werden zur Zusammenarbeit angehalten.

$\checkmark$ Die Vereinten Nationen sollen die einschlägigen Anstrengungen koordinieren.

Bilanz und Position der Schweiz

Die von BUWAL-Direktor Philippe Roch geleitete schweizerische Delegation bestand aus Vertretern des BUWAL, der DEZA, des Bundesamtes für Wasser und Geologie (BWG) sowie aus zwei Vertretern der Arbeitsgemeinschaft der Hilfswerke $^{38}$. Die schweizerische Verhandlungsdelegation sagte in ihrer Bewertung der Konferenz, die Ministererklärung bilde eine gute Grundlage für eine internationale Wasserpolitik, sei aber stärker auf Konsens als auf Innovationen ausgerichtet $^{39}$. Die diesbezügliche Enttäuschung der Schweizer Delegation geht

37 Weltwasserrat, Conclusion du $3^{e}$ Forum mondial de l'eau. 100 nouveaux engagements ont été pris, Pressemitteilung, 23. März 2003.

38 Die Arbeitsgemeinschaft formulierte mehrere Forderungen an die Adresse der Schweizer Delegation. So wird die Delegation gebeten, den Appell für den Schutz des Wassers als öffentliches Gut zu unterstützen. Ausserdem verlangte die Arbeitsgemeinschaft von der Schweiz, sich für ein internationales Wasserübereinkommen einzusetzen (Pressemitteilung, März 2003).

39 „Le Forum mondial de l'eau s'achève sur des propositions ambitieuses“, Le Temps, 24. März 2003; „Le Forum mondial de l'eau n'apporte pas de solutions concrètes au problème“, Le Courrier, 24. März 2003; „Nachhaltigkeit beim Umgang mit Wasser“, Neue Zürcher Zeitung, 18. März 2003; „Wenig Griffiges zur globalen Wassernot“", Neue Zürcher Zeitung, 24. März 2003. 
vor allem darauf zurück, dass die Konferenz es ablehnte, zwei schweizerische Vorschläge in die Abschlusserklärung aufzunehmen. Zunächst beantragte die Schweiz, Wasser als gemeinsames Gut der ganzen Menschheit und den Zugang zu Wasser als Grundrecht anzuerkennen, um eine übermässige Privatisierung der Trinkwasserversorgung $\mathrm{zu}$ vermeiden ${ }^{40}$. Diese Idee setzte sich jedoch nicht durch. Ebenso wenig wurde das von der Schweiz verfochtene „Verursacherprinzip“ in die Ministererklärung aufgenommen. Anlässlich des vorhergehenden Weltforums hatte die Schweiz vorgeschlagen, eine Haftpflichtklausel einzuführen: So hätten die Regierungen die Unternehmen verpflichten können, sich gegen die Risiken der Gewässerverschmutzung zu versichern ${ }^{41}$. Dagegen wird der von der Schweiz befürwortete Ansatz zur Versorgung des Planeten mit Trinkwasser - über den Schutz der Wälder, der Böden und der Feuchtgebiete, welche das Wasser auffangen, filtern, zurückhalten und schliesslich als hochwertiges Trinkwasser wieder freigeben - in der Schlusserklärung verankert ${ }^{42}$.

\section{$\square$ World Water Actions Report}

(Bericht über die weltweiten Aktionen für das Wasser)

Der World Water Actions Report ${ }^{43}$ enthält ein Inventar und eine Analyse der im Zeitraum 2000 bis 2003 weltweit durchgeführten 3000 Aktionen für das Wasser. Der Bericht stellt eine breite Mobilisierung für das Thema Wasser fest, zeigt aber auch die grossen Defizite und die erheblichen Anstrengungen auf, die noch $\mathrm{zu}$ leisten sind, um die Wasserversorgung für alle zu verwirklichen.

Die Hauptempfehlungen des Berichts beziehen sich auf die drei folgenden Ziele ${ }^{44}$ :

- Dezentralisierung der Wasserbewirtschaftung. Die Staaten müssen eine echte Dezentralisierungspolitik durchführen, um nicht nur die begünstigten Regionen, sondern alle Gebiete mit Wasser zu versorgen. Dazu müssen die Mittel und Kompetenzen der Akteure vor Ort gestärkt werden. $\mathrm{Zu}$ den Akteuren zählen auch die Gebietskörperschaften, welche eine zentrale Rolle spielen und die Partner der Zivilgesellschaft und der Privatwirtschaft an der Entwicklung effizienter Wasserdienste beteiligen sollen.

- Finanzielle Mittel. Die finanziellen Investitionen müssen auf allen Ebenen internationale und multilaterale Institutionen, Organe der Vereinten Nationen, Gebietskörperschaften, Regionen und Städte - deutlich erhöht werden.

- Auswertungssystem. Anhand eines Kontroll- und Auswertungssystems sollen die Fortschritte regelmässig evaluiert und der Bedarf vor Ort ermittelt werden. Das System, das von den öffentlichen Behörden unabhängig ist, soll eine Warnfunktion besitzen und gleichzeitig Anreize schaffen.

Die Schlussfolgerungen und die Prioritäten des Berichts wurden anlässlich des 3. Weltwasserforums vorgestellt und erörtert.

40 „La Suisse propose un code de conduite pour réglementer la privatisation de l'eau“, Le Temps, 20. März 2003.

41 „Une idée suisse pour Kyoto“, Le Courrier, 18. März 2003.

42 BUWAL, Kyoto: Ökosysteme für Wasser, Wasser für die Menschen, Medienmitteilung, 22. März 2003.

43 Der Bericht ist auf der Website des Weltwasserrates online einsehbar: <www.worldwatercouncil.org/ download/WWA_Sommaire.pdf>.

44 Weltwasserrat, Publication du rapport „Les actions pour l'eau dans le monde“, Pressemitteilung, 10. März 2003. 


\section{$\square$ Alternatives Weltwasserforum von Florenz}

Das Wasserforum von Florenz tagte Ende März 2003 als Alternative zum offiziellen Forum von Kyoto. Im Mittelpunkt der Diskussionen standen ein Plädoyer für das Recht auf Trinkwasser sowie verschiedene Oppositionsstrategien gegen den Abbau der öffentlichen Dienstleistungen, welche die generelle Wasserversorgung gewährleisten. Ziel des Ersten Alternativen Weltwasserforums war es, allen Bürgern der Welt Zugang zu Trinkwasser zu gewährleisten und Wasser als gemeinsames Gut und gemeinsames Erbe der Menschheit anzuerkennen. Die Ziele leiten sich direkt aus den im Manifest für den Globalen Wasservertrag niedergelegten Grundsätzen ab.

Das Alternative Weltwasserforum ${ }^{45}$ setzte sich die folgenden Ziele:

$\checkmark$ Eine Reihe gesetzgeberischer, politisch-institutioneller, finanzieller und wirtschaftlicher, sozialer und kultureller Massnahmen auf globaler, kontinentaler, nationaler und lokaler Ebene vorschlagen, um das Recht auf Wasser für alle zu verwirklichen - nach einer ganzheitlichen Sicht der Wasserbewirtschaftung und des Wasserschutzes, welche den zeitlichen und räumlichen Zyklen Rechnung trägt.

- Spezifische Kampagnen fördern, um dem allgemeinen Recht auf Wasser und der Anerkennung des Wassers als gemeinsames Gut im Dialog mit den lokalen Gemeinschaften sowie den direktdemokratischen Institutionen Geltung zu verschaffen.

- Synergien erschliessen unter sozialen Bewegungen, Vereinigungen und Organisationen der Zivilgesellschaft, welche in den Bereichen Wasser, Umwelt, Demokratie, Menschenrechte und soziale Rechte tätig sind.

$\checkmark$ Verantwortungsvolles Verhalten der Gesellschaft und des Einzelnen fördern und die Bürger an der Definition und Einhaltung der Vorschriften für den Umgang mit Wasser beteiligen.

\section{$\square$ Aktionen auf Schweizer Ebene}

An die hundert Schweizer Städte beteiligten sich mit konkreten Aktionen am Internationalen Wasserjahr. Sie wurden dabei vom Bundesamt für Wasser und Geologie (BWG), vom Bundesamt für Umwelt, Wald und Landschaft (BUWAL) sowie von der Direktion für Entwicklung und Zusammenarbeit (DEZA) unterstützt. Die schweizerischen Aktionen konzentrierten sich auf drei Schwerpunktthemen: erstens den Schutz der Ökosysteme als Wasserquellen (Wasser ist wertvoll), zweitens den Schutz der Bevölkerung vor den Gefahren des Wassers (Wasser ist unberechenbar) und drittens die Verantwortung der Schweiz als Wasserschloss Europas (Wasser kennt keine Grenzen). Rund zwanzig Kantone beteiligten sich am Projekt. Daneben wurden Aktionen in den Gemeinden und in Schulen durchgeführt (Ausstellungen, Konferenzen usw.) ${ }^{46}$.

45 Website des Forums : <www.cipsi.it/contrattoacqua/forum-acqua/index.htm>.

46 „Quarante projets en Suisse pour rappeler l'importance de l'eau“, Le Courrier, 22. März 2003. 


\section{QUELLEN}

Weltwasserrat, Conclusion du $3^{e}$ Forum mondial de l'eau. 100 nouveaux engagements ont été pris, Pressemitteilung, 23. März 2003.

Weltwasserrat, Déclaration ministérielle du 3e Forum mondial de l'eau, 23. März 2003.

Weltwasserrat, Publication du rapport „Les actions pour l'eau dans le monde“, Pressemitteilung, 10. März 2003.

Weltwasserrat, ,3 $3^{\text {rd }}$ World Water Council: Summary“, Forum Bulletin, März 2003.

Weltwasserrat, Japan Water Resources Association, UNESCO, Les actions pour l'eau dans le monde, Marseille, 2003, einsehbar unter : <www.worldwatercouncil.org/download/WWA_Sommaire.pdf>.

BUWAL, Kyoto: Ökosysteme für Wasser, Wasser für die Menschen, Pressemitteilung, 22. März 2003.

Le Courrier, „Un creuset de bonne volonté pour le droit à l'eau pour tous“, 17. März 2003; „Au Ghana, l'eau est confisquée par la logique du marché“, 22. März 2003; „Quarante projets en Suisse pour rappeler l'importance de l'eau“, 22. März 2003; „Le Forum mondial de l'eau n'apporte pas de solutions concrètes au problème“, 24. März 2003.

Le Temps, „La Suisse propose un code de conduite pour réglementer la privatisation de l’eau“, 20. März 2003 ; „Le Forum mondial de l'eau s'achève sur des propositions ambitieuses“, 24. März 2003.

Neue Zürcher Zeitung, „Kampf um Wasser als globales Politikum“, 17. März 2003; „Nachhaltigkeit beim Umgang mit Wasser“, 18. März 2003; „Wenig Griffiges zur globalen Wassernot“, 24. März 2003.

\section{INTERNET-ADRESSEN}

Internationales Wasserjahr, offizielle Website: <www.wateryear2003.org>.

Weltwasserrat: <www.worldwatercouncil.org $>$.

3. Weltwasserforum: <www.world.water-forum3.com>.

Arbeitsgemeinschaft der Hilfswerke - Themen „Wasser“: <www.swisscoalition.ch>.

Alternativer Wassergipfel : <www.cipsi.it/contrattoacqua/forum-acqua/index.htm>.

\subsection{Andere Entwicklungen im Umweltbereich}

\section{$\square$ Handel und Umgang mit giftigen Stoffen und Abfällen}

Vier wichtige multilaterale Instrumente regeln den Handel und Umgang mit giftigen Chemikalien und Abfällen. Es sind dies die Konvention von Basel und ihre Änderung (1989/1995) über den Handel, die Entsorgung und die Minimierung gefährlicher Abfälle; das Protokoll zur Konvention von London zum Schutz der Meere vor der Versenkung von Abfällen (1996); die Konvention von Rotterdam (PIC-Konvention 1998) über das Verfahren der vorherigen Zustimmung nach Inkenntnissetzung für bestimmte gefährliche Chemikalien und Pestizide im internationalen Handel sowie die Konvention von Stockholm (POP-Konvention 2001) über das Verbot persistenter organischer Schadstoffe. Bis Ende 2003 konnte mangels einer genügenden Anzahl Ratifikationen noch kein Abkommen in Kraft treten ${ }^{47}$. Für die beiden letztgenannten Konventionen ist aber ein InKraft-Treten im Jahr 2004 wahrscheinlich.

In der Schweiz war die Botschaft des Bundesrates zur Ratifizierung der POPKonvention unumstritten. Nach Zustimmung beider Räte konnte die Konvention im Juli 2003 ratifiziert werden. Damit gehört die Schweiz mit Schweden, Norwegen und Deutschland zu den Ländern, welche alle vier Abkommen ratifiziert haben ${ }^{48}$.

Jahrbuch 2003, Nr. 1, in Kap. 10.5, „Handel und Umgang mit giftigen Chemikalien“, S. 200-203.

47 Die Konvention ist in Kraft getreten, nicht aber ihre Änderung von 1995.

48 Noch keine der Konventionen ratifiziert haben Brasilien, Frankreich, Indien, Russland und die USA. 
Ausgediente Computer und anderes elektronisches Büromaterial stellen mit einer Wachstumsrate von bis zu 5 Prozent weltweit die zur Zeit am schnellsten wachsende Abfallart dar ${ }^{49}$. Ein beträchtlicher Teil dieses teilweise hochgiftigen Elektronikschrottes ${ }^{50}$ (e-waste) wird auf Müllhalden entsorgt oder unsachgemäss rezykliert ${ }^{51}$. Die Basler Konvention soll verhindern, dass Sondermüll aus den Industriestaaten in arme Ländern exportiert wird. Doch Länder wie die USA, Japan, Frankreich oder Kanada haben bis heute eine Ratifizierung verweigert und verhindern damit ein In-Kraft-Treten der Konvention. Experten schätzen, dass diese Länder bis zu 80 Prozent ihrer Elektronikabfälle in Länder wie China, Indien und Pakistan exportieren. Bei der dortigen Verarbeitung des Computerschrotts wird die Umwelt zerstört und die Gesundheit der im Sektor beschäftigten Arbeiterinnen und Arbeiter geschädigt.

Aus diesem Grund möchte die Schweiz ihre Erfahrung im Recycling von Elektronikschrott weitergeben. Ein vom Staatssekretariat für Wirtschaft (seco) finanziertes Forschungsprojekt soll die Gefahren bei der Entsorgung vermindern helfen $^{52}$. Das Teilprojekt mit dem Namen „Knowledge Management and Information Dissemination“ dokumentiert, adaptiert und integriert das weltweit bestehende Wissen im Elektronikschrott-Recycling. Daraus entsteht ein bis zum Sommer 2004 im Internet zugängliches Elektronikschrott-Handbuch. Das Teilprojekt „Capacity Building“ untersucht, ob es möglich ist, in den drei Städten New Dehli, Beijing und Johannesburg ein nachhaltiges Recycling-System einzuführen. In einer nächsten Phase sollen Verbesserungen gemeinsam geplant und umgesetzt werden.

\section{$\checkmark$ Schutz der Ozonschicht}

Vom 10. bis 14. November 2003 trafen sich die 184 Vertragsparteien des Protokolls von Montreal zu ihrer 15. Konferenz in Nairobi ${ }^{53}$. Ziel des Protokolls von Montreal ist es, die Ozonschicht zu erhalten, indem ozonschichtabbauende Gase auf weltweiter Ebene vollständig eliminiert werden. Den Schwerpunkt der Konferenz bildeten Verhandlungen über eine Ausnahmeregelung für den Gebrauch der Substanz Methylbromid. Der in der Landwirtschaft als Schädlingsbekämpfungsmittel eingesetzte Stoff ist 40-mal schädlicher für die Ozonschicht als die Fluorkohlenwasserstoffe. Das Ozonschutzprotokoll verlangt von den Industrieländern einen Ausstieg aus Methylbromid bis 2005, von den Entwicklungsländern bis 2015. Da in der Landwirtschaft noch keine kostengünstigen Alternativen zu Methylbromid zur Verfügung stehen, forderten einige Industrieländer grosszügige Ausnahmeregelungen für den Gebrauch der Substanz. Die Konferenzteilnehmer fanden keinen Konsens und werden das Thema an einem späteren ausserordentlichen Treffen weiterverhandeln.

Beobachter befürchten, dass grosszügige Ausnahmeregeln das Protokoll untergraben und als Präzedenzfall bei weiteren, die Ozonschicht zerstörenden Stoffen dienen könnten.

49 Basel Convention Newsletter, „The toxic legacy of e-waste“: <www.basel.int>, 20. April 2003.

50 Giftige Bestandteile sind unter anderem Arsen, Blei, Brom, Cadmium, PVC und Quecksilber.

51 Neue Zürcher Zeitung, ,Wenn Computer sterben, wird es giftig“, 22. November 2003, und Basel Action Network, ,The Electronics Recycler's Pledge of True Stewardship“: <www.ban.org>, Februar 2003.

52 Eidgenössische Materialprüfungs- und Forschungsanstalt (EMPA), Wissens-Partnerschaft mit Entwicklungs- und Schwellenländern im Recycling von Elektronikschrott (eWaste): <www.empa.ch>.

53 The $15^{\text {th }}$ Meeting of the Parties to the Montreal Protocol : <www.unep.org/ozone/mop/15mop $>$. 
Die Schweiz zahlt jedes Jahr rund 2 Millionen US-Dollar in den multilateralen Fonds des Protokolls von Montreal ein. Hauptzweck des Fonds ist es, die Entwicklungsländer bei ihren Anstrengungen zu unterstützen, innerhalb der gesetzten Fristen auf die Verwendung der ozonschichtabbauenden Stoffe zu verzichten. Weiter unterstützt die Schweiz die Umsetzung des Protokolls mit bilateralen Projekten im Bereich der Kältetechnik.

Am 1. Juli 2003 sind die vom Bundesrat verordneten neuen Bestimmungen über umweltgefährdende Stoffe (Stoffverordnung) in Kraft getreten ${ }^{54}$. Damit sollen der Schutz der Ozonschicht verstärkt und die synthetischen Treibhausgase verringert werden. Die veränderten Bestimmungen verbieten, von gewissen Ausnahmen abgesehen, die Ein- und Ausfuhr, die Herstellung und den Gebrauch einer Reihe von ozonschichtabbauenden Stoffen ${ }^{55}$. Für die vom Verbot nicht betroffenen Mengen wird ein Kontrollsystem zur Ein- und Ausfuhr errichtet. Die Einfuhr von Methylbromid zum Zweck der Schädlingsbekämpfung wurde ab 2003 stark eingeschränkt und wird ab 2005 ganz verboten.

\section{$\square$ Biodiversitätskonvention und Protokoll von Cartagena}

Das im Jahr 2000 im Rahmen der Biodiversitätskonvention verabschiedete und von 72 Ländern ratifizierte Cartagena-Protokoll über die biologische Sicherheit ist am 11. September 2003 in Kraft getreten. Es ist das erste völkerrechtliche Instrument, das sich mit den Aspekten der Sicherheit von Umwelt und Gesundheit im Zusammenhang mit der Verwendung von gentechnisch veränderten Organismen (GVO) befasst. Das Protokoll regelt den internationalen Handel mit lebenden und keimfähigen gentechnisch veränderten Organismen. Vertragsstaaten sind verpflichtet, bei einer erstmaligen Ausfuhr von gentechnisch veränderten Organismen das Einfuhrland zu benachrichtigen. Dem einführenden Land wird der Zugang zu allen Informationen garantiert, welche nötig sind, um die Umweltrisiken im Zusammenhang mit den GVO abzuschätzen. Damit kann das betreffende Land in Kenntnis der Sachlage über eine mögliche Einfuhr entscheiden.

Das Protokoll ist für Entwicklungsländer, die noch über keine nationale Gesetzgebung im Bereich der Biotechnologie verfügen, von besonderer Bedeutung. Ein weiteres Ziel des Protokolls ist die Förderung der wissenschaftlichen und technischen Zusammenarbeit zwischen Nord und Süd. Mit dem Biosafety Clearing House wird ein weltweites System zur Information über die Risikominderung im Biotechnologiebereich aufgebaut ${ }^{56}$.

Das Protokoll fordert auch die Schaffung nationaler Anlaufstellen (National Focal Points), welche damit beauftragt sind, die innerstaatlichen Bestimmungen über die Ausfuhr von lebenden veränderten Organismen umzusetzen, die Massnahmen im Bereich des Informationsausstausches zu koordinieren und die Koordination mit der internationalen Ebene zu gewährleisten ${ }^{57}$.

54 Medienmitteilung BUWAL, Synthetische Treibhausgase unter Kontrolle, Schutz der Ozonschicht verstärkt, Bern, 30. April 2003.

55 Es handelt sich um die Substanzen FCKW, Halone, Tetrachlorkohlenstoff, Trichlorethan und Bromchlormethan.

56 Pilotphase des Biosafety Clearing-House: <http://bch.biodiv.org/Pilot/Home.aspx>.

57 Die Schweiz hat das Protokoll im März 2002 ratifiziert. Ihre Informationsplattform für biologische Sicherheit (Swiss Biosafety Clearing-House) befindet sich noch in der Pilotphase: <www.ch-bch.ch>. 
Am 1. Januar 2004 ist in der Schweiz das im März 2003 vom Parlament verabschiedete Gentechnikgesetz in Kraft getreten ${ }^{58}$. Ziel des neuen Gesetzes ist der Schutz von Mensch und Umwelt vor Missbräuchen der Gentechnologie ${ }^{59}$. Geregelt wird unter anderem der Schutz der gentechnikfreien Produktion, die Wahlfreiheit der Konsumenten und die eindeutige Kennzeichnung von gentechnisch veränderten Produkten. Die Haftpflicht wurde verschärft und bringt im Vergleich zu früher längere Verjährungsfristen sowie die Abdeckung von Umweltschäden. Wer versuchsweise gentechnisch veränderte Organismen freisetzen will, muss gemäss dem geänderten Umweltgesetz aus Gründen der Biosicherheit im Vergleich zu früher erhöhte Anforderungen erfüllen.

Jahrbuch 2003, Nr. 1, Kap. 10.4., „Umsetzung der Biodiversitätskonvention“, S. 190-196.

\section{$\square$ Konvention zur Bekämpfung der Wüstenbildung}

Vom 25. August bis 6. September 2003 trafen sich die Mitgliedsländer der Konvention zur Bekämpfung der Wüstenbildung (UNCCD) zu ihrer 6. Vertragsparteienkonferenz in Havanna ${ }^{60}$. Die Konferenz war vom Übergang der Phase der Bewusstseinsbildung und Planung in die Phase der konkreten Umsetzung geprägt. Zeichen dieser neuen Phase war die Bestimmung des Globalen Umweltfonds (GEF) als Finanzierungsmechanismus der Konvention. Für die nächsten drei Jahre stellt der Fonds 500 Millionen US-Dollar zur Verfügung. Auch wenn diese Summe allein nicht ausreicht, um die Wüstenbildung aufzuhalten, kann sie doch die Umsetzung der Konvention fördern ${ }^{61}$. Zudem erfährt die Konvention durch die Unterstützung des GEF eine Aufwertung gegenüber anderen Umweltkonventionen (Klima, Ozon, Biodiversität), welche oftmals als bedeutender eingestuft werden.

Die Teilnehmer betonten die Wichtigkeit der Konvention für die Armutsbekämpfung und riefen namentlich die Erdöl exportierenden arabischen Staaten auf, sich finanziell vermehrt an den internationalen Bestrebungen für den Bodenschutz zu beteiligen. Einige Geberländer machten ihre Unterstützung für die Konvention von einem transparenteren und effizienteren Sekretariat abhängig.

Auf der Konferenz wurde ferner an der Nutzung der Synergien zwischen den aus dem Erdgipfel von Rio hervorgegangenen drei Konventionen über Wüstenbildung, Klima und Biodiversität gearbeitet.

Die Schweiz unterstützt den Kampf gegen die Wüstenbildung durch ihre finanzielle Beteiligung am Globalen Umweltfonds und mit bilateralen Projekten in der Sahelregion sowie in Trockengebieten der Anden und Zentralasiens. Die schweizerische Federführung für die UNO-Konvention zur Bekämpfung der Wüstenbildung liegt bei der DEZA. la Sixième Conférence des Parties de la Convention pour la Lutte contre la Désertification, 8. September 2003 : <www.iisd.ca>; Sixth Session of the Parties : <www.unccd.int/cop/cop6/menu.php>.

61 Die jährlichen Kosten für die Umsetzung der Konvention werden auf 2,4 Milliarden US-Dollar, die jährlichen Verluste durch Wüstenbildung auf 42 Milliarden US-Dollar geschätzt. 


\section{Umweltstrategie für Osteuropa und Zentralasien}

Vom 21. bis 23. Mai 2003 fand die fünfte gesamteuropäische Ministerkonferenz „Umwelt für Europa“ in Kiew statt ${ }^{62}$. Teilnehmer waren die 55 Mitgliedsstaaten der UNO-Wirtschaftskommission für Europa, darunter die USA, Kanada sowie 25 Transitions- und Entwicklungsländer. Das Ziel des Prozesses „Umwelt für Europa“ ist die Förderung der Zusammenarbeit der europäischen Staaten im Umweltbereich und die Erhöhung der Umweltstandards in den Ländern des ehemaligen Ostblocks. Im Zuge der EU-Osterweiterung und der Verschärfung der Umweltbestimmungen in den Beitrittsländern verlagerten sich die Aktivitäten in die Länder der Gemeinschaft Unabhängiger Staaten (GUS) und nach Südosteuropa.

Die europäische Umweltkonferenz verabschiedete eine Strategie für die Umweltzusammenarbeit der zwölf GUS-Staaten. Die Strategie stellt eine gemeinsame Basis für die Lösung von Umweltproblemen in den Bereichen Wasser, gefährliche Abfälle und Biodiversität dar. In einer Schlussdeklaration unterstrichen die Minister ihren Willen, den Verlust der biologischen Vielfalt bis zum Jahr $2010 \mathrm{zu}$ stoppen, und forderten die UNO-Wirtschaftskommission für Europa auf, zum Bindeglied zwischen der regionalen Ebene und dem weltweiten Prozess der nachhaltigen Entwicklung zu werden ${ }^{63}$.

Anlässlich der Konferenz standen drei Protokolle zur Unterzeichnung bereit. Das Protokoll über die Haftpflicht regelt den Schadenersatz bei grenzüberschreitenden Schäden infolge von Industrieunfällen. Das Protokoll über die strategische Umweltprüfung sieht vor, Umweltanliegen bereits auf der strategischen Entscheidungsstufe der Behörden zu berücksichtigen und damit gleichzeitig die Umweltprüfung auf der Projektstufe zu entlasten. Das dritte Protokoll über Emissions- und Transferregister von Schadstoffen verbessert den Informationszugang der Öffentlichkeit durch den Aufbau nationaler Register mit Daten zu 86 Umweltschadstoffen.

Was die Umwelt betrifft, so engagiert sich die Schweiz in Osteuropa hauptsächlich in den Bereichen der Finanzierung von Massnahmen zur Erhaltung der Artenvielfalt, der Wasserwirtschaft und dem Schutz von Ökosystemen ${ }^{64}$. Namentlich unterstützt die Schweiz die „Europäische Initiative zur Finanzierung der Biodiversität mit Hilfe privater Partner" und die strategische Partnerschaft „Wasser für nachhaltige Entwicklung“.

Im Juni 2003 nahm die Schweizer Stimmrechtsgruppe im Globalen Umweltfonds Kasachstan als neues Mitglied auf ${ }^{65}$. Die Ländergruppe umfasst ausser der Schweiz die Staaten Aserbeidschan, Kirgisistan, Tadschikistan, Turkmenistan und Usbekistan. Mit dem Einbezug Kasachstans soll in erster Linie die Zusammenarbeit zwischen den zentralasiatischen Staaten bei der Lösung von Umweltproblemen gestärkt werden.

62 „Environment for Europe“ Process : <www.unece.org/env/wgso/index_kyivconf.htm>; Fünfte Ministerkonferenz „Umwelt für Europa“ <www.kyiv-2003.info>; Pressemitteilung BUWAL, 14. Mai 2003, 23. Mai 2003; BUWAL, Umwelt 1/03, „Ökologischer Marschallplan für den Osten“, Bern, 2003.

63 Fifth Ministerial Conference „Environment for Europe“ Declaration by the Environment Ministers of the Region of the United Nations Economic Commission for Europe (UNECE), Kiew, 23. Mai 2003.

64 BUWAL Faktenblatt, Umweltkooperation mit den GUS-Staaten, Bern, Mai 2003,

65 Pressemitteilung BUWAL, Globaler Umweltfonds GEF, 26. Juni 2003. 


\section{QUELLEN}

BUWAL, Umwelt Schweiz, Band 1 und 2, Bern, 2002.

Institut International du Développement Durable, Bulletin de Négociations de la Terre, Résumée de la Sixième Conférence des Parties de la Convention pour la Lutte contre la Désertification, 8. September 2003 .

International Institute for Sustainable Development, Earth Negotiations Bulletin, Summary of the fifteenth Meeting of the Parties to the Montreal Protocol, November 2003.

\section{INTERNET-ADRESSEN}

Basel Action Network: <www.ban.org >.

Bundesamt für Umwelt Wald und Landschaft (BUWAL) : <www.buwal.ch>.

Informationsplattform der Schweiz zur Umsetzung der Konvention über die biologische Vielfalt von Rio 1992 (Schweizerischer Vermittlungsmechanismus Biodiversität) : <www.ch-chm.ch $>$.

International Institute for Sustainable Development (IISD) : <www.iisd.org $>$.

Ozon-Sekretariat: <www.unep.org/ozone>.

PIC-Konvention: <www.pic.int>.

Pilotphase des Biosafety Clearing-House : <http://bch.biodiv.org/Pilot/Home.aspx>.

Secretariat of the United Nations Convention to Combat Desertification: <www.unccd.int $>$.

POP-Konvention: <www.pops.int>.

Sekretariat der Basler Konvention: <www.basel.int>.

United Nations Economic Commission for Europe (UNECE) : <www.unece.org $>$.

Übereinkommen über die biologische Vielfalt: $<w w w . b i o d i v . o r g>$. 